\title{
Biphasic Regulation of p38 MAPK by Serotonin Contributes to the Efficacy of Stimulus Protocols That Induce Long-Term Synaptic Facilitation
}

\author{
(D)Yili Zhang, Paul Smolen, Douglas A. Baxter, and Johohn H. Byrne
}

DOI:http://dx.doi.org/10.1523/ENEURO.0373-16.2017

Department of Neurobiology and Anatomy, W.M. Keck Center for the Neurobiology of Learning and Memory, McGovern Medical School, University of Texas Health Science Center at Houston, Houston, TX 77030

\begin{abstract}
The MAPK isoforms ERK and p38 MAPK are believed to play opposing roles in long-term synaptic facilitation (LTF) induced by serotonin (5-HT) in Aplysia. To fully understand their roles, however, it is necessary to consider the dynamics of ERK and p38 MAPK activation. Previous studies determined that activation of ERK occurred $\sim 45$ min after a 5-min pulse of 5-HT treatment. The dynamics of p38 MAPK activation following 5-HT are yet to be elucidated. Here, the activity of p38 MAPK was examined at different times after 5-HT, and the interaction between the ERK and p38 MAPK pathways was investigated. A 5-min pulse of 5-HT induced a transient inhibition of p38 MAPK, followed by a delayed activation between 25 and $45 \mathrm{~min}$. This activation was blocked by a MAPK kinase inhibitor, suggesting that similar pathways are involved in activation of ERK and p38 MAPK. ERK activity decreased shortly after the activation of p38 MAPK. A p38 MAPK inhibitor blocked this decrease in ERK activity, suggesting a causal relationship. The p38 MAPK activity $\sim 45 \mathrm{~min}$ after different stimulus protocols was also characterized. These data were incorporated into a computational model for the induction of LTF. Simulations and empirical data suggest that p38 MAPK, together with ERK, contributes to the efficacy of spaced stimulus protocols to induce LTF, a correlate of long-term memory (LTM). For example, decreased p38 MAPK activity $\sim 45$ min after the first of two sensitizing stimuli might be an important determinant of an optimal interstimulus interval (ISI) for LTF induction.
\end{abstract}

Key words: biphasic regulation; computational model; ERK; LTF; p38 MAPK; spaced stimulation

\section{Significance Statement}

MAPK pathways play critical roles in mediating diverse forms of synaptic plasticity. Activation of the ERK isoform is required for long-term synaptic facilitation (LTF), whereas the p38 MAPK isoform is required for long-term synaptic depression (LTD). Here, we used isolated Aplysia sensory neurons (SNs) to confirm and extend previous studies delineating dynamics of ERK and p38 MAPK. We quantified p38 MAPK activity after application of the essential neurotransmitter 5-HT and explored the cross talk between p38 MAPK and ERK in SNs. These data were incorporated into a computational model for the induction of LTF. Simulations suggest that p38 MAPK, together with ERK, contributes to the efficacy of temporally spaced stimulus protocols to induce LTF, a correlate of long-term memory (LTM).

\section{Introduction}

MAPK pathways play critical roles in mediating diverse forms of synaptic plasticity (English and Sweatt, 1997; Adams and Sweatt, 2002; Hu et al., 2004). The interac-

Received December 15, 2016; accepted January 19, 2017; First published January 26, 2017.

The authors declare no competing financial interests. tions among MAPK isoforms are believed to regulate the nature (increases vs decreases) and duration (short- vs long-term) changes in synaptic strength. For example, the sensorimotor synapse in Aplysia exhibits at least two 
forms of long-term plasticity. Long-term synaptic facilitation (LTF) can be induced by repeated application of the neuromodulator serotonin (5-HT) (Schacher et al., 1990, 2000), whereas long-term synaptic depression (LTD) can be induced by application of the peptide Phe-Met-ArgPhe-NH2 (FMRFa) (Montarolo et al., 1988; Schacher et al., 2000; Guan et al., 2002). Activation of the ERK isoform is required for LTF (Martin et al., 1997; Michael et al., 1998; Purcell et al., 2003; Ormond et al., 2004; Sharma and Carew, 2004), whereas activation of the p38 kinase isoform is required for LTD (Guan et al., 2002, 2003). What is less clear, however, are the ways in which these two opposing MAPK pathways are regulated and how interactions between the ERK and p38 kinase pathways lead to a specific outcome (e.g., LTF vs LTD). Guan et al. (2002, 2003) proposed a model in which the two pathways are regulated by different inputs and reciprocally inhibit one another. For example, they suggested that 5-HT-induced activation of protein kinase $A$ (PKA), which is necessary for LTF, may in turn inhibit p38 MAPK, thereby further supporting LTF. Here, we investigate an alternative but not mutually exclusive hypothesis in which dynamics of ERK and p38 MAPK activation determine the outcome of plasticity mediated by MAPK pathways. 5-HT activation of ERK leads to inactivation of the transcription repressor CREB2 (Bartsch et al., 1995). It is likely that ERK also activates the transcription activator CREB1, via activation of the CREB kinase denoted ribosomal S6 kinase (Choi et al., 2011; Philips et al., 2013b). In contrast, p38 MAPK mediates LTD by activating CREB2 and enhancing CREB2-mediated repression of genes such as c/ebp (Guan et al., 2002; 2003). Moreover, the 5-HT and FMRFa pathways interact at the levels of ERK and p38 MAPK. 5-HT inhibits p38 MAPK activity and activates ERK, whereas FMRFa activates p38 MAPK and inhibits ERK (Guan et al., 2003; Fioravante et al., 2006). Consequently, a plausible hypothesis is that the dynamic balance of these MAPK isoforms determines the direction of synaptic plasticity.

This hypothesis is based, in part, on previous observations that the dynamics of ERK activation are complex (Ye et al., 2008; Philips et al., 2013b; see also Ajay and Bhalla, 2004). For example, in sensorimotor synapses, a single 5 -min pulse of $50 \mu \mathrm{M} 5-\mathrm{HT}$ has no effect on ERK phosphorylation and, thus, activation when examined immediately after treatment. However, the 5-HT pulse induces a delayed activation of ERK $\sim 45$ min after treatment. In contrast, Guan et al. (2003) reported that phosphorylation and thus activation of p38 MAPK is reduced immediately after a 10-min treatment of $50 \mu \mathrm{M} 5-\mathrm{HT}$. Currently, noth-

\footnotetext{
contributed to all aspects of these studies. All authors discussed the results and contributed to the writing and editing of the manuscript.

This work was supported by NIH Grants NS019895 and NS073974.

Correspondence should be addressed to John H. Byrne, Ph.D., Department of Neurobiology and Anatomy, W.M. Keck Center for the Neurobiology of Learning and Memory, McGovern Medical School, Houston, TX 77030. E-mail: John.H.Byrne@uth.tmc.edu.

DOI:http://dx.doi.org/10.1523/ENEURO.0373-16.2017

Copyright (C) 2017 Zhang et al.

This is an open-access article distributed under the terms of the Creative Commons Attribution 4.0 International, which permits unrestricted use, distribution and reproduction in any medium provided that the original work is properly attributed.
}

ing is known about the subsequent time course of p38 MAPK phosphorylation. Philips et al. (2013b) also found that ERK activity, elevated at $45 \mathrm{~min}$, returns to control level at 60 min after a 5-min pulse of $50 \mu \mathrm{M} 5-\mathrm{HT}$. It is not evident how ERK activity can be downregulated to control level within $\sim 15 \mathrm{~min}$.

Here, we used isolated Aplysia sensory neurons (SNs) to confirm and extend the previous studies in dynamics of ERK and p38 MAPK. We quantified the dynamics of MAPK activity after a 5 -min pulse of $5-\mathrm{HT}(50 \mu \mathrm{M})$ and explored the cross talk between p38 MAPK and ERK pathways in SNs.

It is commonly accepted that spaced stimulus protocols (i.e., with long intervals between sessions) are more efficient in inducing LTF and long-term memory (LTM) than are massed protocols (i.e., short or no intervals between sessions) (Mauelshagen et al., 1998; Philips et al., 2013a; Smolen et al., 2016). Philips et al. (2007; 2013b) similarly found that two tail shocks to Aplysia could induce long-term sensitization of a withdrawal reflex, a form of LTM, but only when separated by 45 min. No LTM was observed with an interstimulus interval (ISI) that was too short (15 min) or too long (60 min). Simulations with a computational model we developed suggest that the ISI of 45 min is superior in inducing LTM, because the ratio of ERK to p38 MAPK activity is maximized by an ISI of $\sim 45$ min, mainly due to a delayed decrease in p38 MAPK activity at this time.

\section{Materials and Methods}

\section{Neuronal cultures}

This study used juvenile Aplysia, which are hermaphroditic invertebrates. Aplysia californica (80-100 g) were supplied by The University of Miami and maintained in circulating artificial seawater at $15^{\circ} \mathrm{C}$. SNs were isolated from the ventral-caudal cluster of the pleural ganglion from Aplysia. Before extraction of ganglia, animals were anesthetized by injection of isotonic $\mathrm{MgCl}_{2}$ equal to approximately one half of the body volume. Ganglia were removed and incubated in culture medium containing $50 \%$ hemolymph and $50 \%$ isotonic L15 at room temperature and then manually desheathed. SNs were removed from the ganglia individually by microelectrodes with fine tips and plated on poly-L-lysine-coated glass slides in petri dishes of culture medium. Dishes of SN cultures were plated with four to eight SNs and allowed to grow for $5 \mathrm{~d}$ at $18^{\circ} \mathrm{C}$ before experiments were begun.

\section{Pharmacological treatment}

Dishes of SNs cultured from the same animals were paired for all the 5-HT treatments. In each pair, one dish received solution which consisted of $50 \%$ isotonic L15 and 50\% artificial seawater (L15/ASW) (ASW: $450 \mathrm{mM}$ $\mathrm{NaCl}, 10 \mathrm{mM} \mathrm{KCl}, 11 \mathrm{mM} \mathrm{CaCl}_{2}, 29 \mathrm{mM} \mathrm{MgCl}_{2}$, and 10 $\mathrm{mM}$ HEPES, $\mathrm{pH}$ 7.6) as vehicle control (Veh). The other received the same solution with the addition of $50 \mu \mathrm{M}$ 5-HT. The experimenter was blind to the identity of the treatments. In the experiments to measure the time course of phosphorylated ERK (pERK) or phosphorylated p38 MAPK ( $p-p 38$ MAPK) after one pulse of $5-H T$, if four 
pairs of dishes were available, one dish was fixed immediately after wash off of 5-HT for immunofluorescence (see below). The other three dishes were incubated in L15/ASW after wash off of 5-HT, until they were fixed at 15,45 , and 60 min after onset of 5-HT for immunofluorescence. The remaining four dishes served as timematched Veh controls. For each pair of dishes measured at the same time point, the averaged pERK or p-p38 MAPK from the dish receiving $5-\mathrm{HT}$ was compared with the averaged pERK or p-p38 MAPK from the Veh control. If two to three pairs were available, one pair was fixed immediately after wash off of 5-HT, the others were fixed at one or two of the above later times. When two pulses of $5-\mathrm{HT}$ were given to SNs, the ISI was defined as the interval from the onset of the first stimulus to the onset of the second stimulus. During the ISI, SNs were incubated in L15/ASW after wash out of 5-HT.

To examine the effects of $\mathrm{p}$-p38 MAPK on pERK, $3 \mu \mathrm{M}$ SB203580 (EMD Millipore) was applied to SN cultures 30 min before $5-\mathrm{HT}$ treatments (one 5 -min pulse of $50 \mu \mathrm{M}$ $5-\mathrm{HT}$ ) and then concurrently with $5 \mathrm{~min} 5-\mathrm{HT}$ treatment. At this concentration ( $3 \mu \mathrm{M}$ ) SB203580 blocks LTD in Aplysia without affecting basal synaptic strength (Guan et al., 2003). Four dishes of SNs from the same animals were used for each experiment. Each dish was given a different treatment, either (1) $50 \mu \mathrm{M} 5-\mathrm{HT}$ alone, (2) $3 \mu \mathrm{M}$ SB203580 alone, (3) 5-HT + SB203580, or (4) Veh alone. After 5-HT was washed out, treatment with SB203580 was continued until fixation for immunofluorescence.

To examine the effect of MEK1/2 inhibition on p-p38 MAPK, $20 \mu \mathrm{M}$ U0126 (Promega) was applied to SN cultures $30 \mathrm{~min}$ before and then concurrently with 5 -min 50 $\mu \mathrm{M} 5-\mathrm{HT}$ treatment. At this concentration $(20 \mu \mathrm{M}), \mathrm{U} 0126$ blocks ERK phosphorylation after 5-HT treatment in Aplysia (Sharma et al., 2003). Four dishes of SNs from the same animals were used for each experiment and given different treatments, either (1) $50 \mu \mathrm{M} \mathrm{5-HT}$ alone, (2) 20 $\mu \mathrm{M}$ U0126 alone, (3) 5-HT + U0126, or (4) Veh alone. After 5 -HT was washed out, U0126 treatment was continued until fixation for immunofluorescence.

\section{Immunofluorescence}

Immunofluorescence procedures for SNs followed those of Liu et al. (2014). Briefly, at different time points after the onset of 5-HT treatment, cells were fixed in a solution of $4 \%$ paraformaldehyde in PBS containing $20 \%$ sucrose. After three 5-min rinses in PBS, fixed cells were blocked for $30 \mathrm{~min}$ at room temperature in a solution of Superblock buffer (Pierce), 0.2\% Triton X-100 and 3\% normal goat serum, and subsequently incubated overnight at $4^{\circ} \mathrm{C}$ with anti-pERK rabbit antibody (1:200), or anti-p-p38 MAPK rabbit antibody (1:200). Anti-pERK antibodies [phospho-p44/42 MAPK (Erk1/2) XP Rabbit mAb, catalog \#4370S; RRID: AB2315112] and anti-p-p38 MAPK antibodies [phospho-p38 MAPK (Thr180/Tyr182) XP Rabbit mAb, catalog \#4511S; RRID: AB2139682] were purchased from Cell Signaling Technology. Secondary antibody (goat anti-rabbit secondary antibody conjugated to Rhodamine Red; 1:200 dilution; Thermo Scientific, catalog \#R-6394) was applied for $1 \mathrm{~h}$ at room temperature.
Cells were then mounted using Mowiol 4-88 (SigmaAldrich). The intensity of staining in SNs was quantified in images obtained with a Zeiss LSM510 confocal microscope using a $63 \times$ oil-immersion lens as described previously (Liu et al., 2014). A z-series of optical sections through the cell body $(0.5-\mu \mathrm{m}$ increments) were taken, and the section through the middle of the nucleus was used for analysis of mean fluorescence intensity with MetaMorph Offline software (Universal Imaging). Four to eight neurons on each coverslip were analyzed, and measurements from neurons on the same coverslip were averaged.

\section{Statistical analyses}

SigmaPlot version 11 (Systat Software) was used. Data are presented by box-and-whisker plots, and $p<0.05$ was considered to represent statistical significance. Before making comparisons between groups, Shapiro--Wilk normality and equal variance tests were performed. For data displaying normal distribution, paired $t$ test was used for comparison between paired Veh and treatment groups, and one-way ANOVA and the post hoc Student-Newman--Keuls method were used for multiple comparisons between groups. For data displaying non-normal distribution, Wilcoxon signed-rank test (WSRT) was used for comparison between paired Veh and treatment groups, and Kruskal--Wallis one-way ANOVA on ranks and the post hoc Student--Newman-Keuls method were used for multiple comparisons between groups.

\section{Model development and equations}

The mathematical model for the activation (i.e., phosphorylation) of ERK and p38 MAPK was modified from a previously published model (Zhang et al., 2012) of the signaling cascades underlying the induction of LTF.

\section{ERK pathway}

The activation of ERK was modeled as a cascade with sequential activation of the kinases Raf, MEK1 (MAPKK), and ERK. The ordinary differential equations describing the activation or phosphorylation of Raf, MEK1, and ERK (Eqs. 1-8) are similar to those in Zhang et al. (2012). To allow simulation of the quantitative changes of pERK by 5-HT, basal kinase activities were added to the equations describing the Raf pathway (Eq. 1). [MEK1] in Eqs. 3-10 represents the MEK1/2 isoforms known to phosphorylate ERK (Shaul and Seger, 2007). [ERK $\left.{ }^{p D}\right]$ corresponds to the pERK level measured by immunofluorescence. As described in Zhang et al. (2012), a discrete time delay ( $\tau_{\text {delay }}$ $=25 \mathrm{~min}$ ) was added to the phosphorylation of Raf in Eq. 1. Because the mechanism underlying the delay in ERK phosphorylation is unknown, $\tau_{\text {delay }}$ is used to ensure that $\mathrm{pERK}$ has a peak at $\sim 45 \mathrm{~min}$ after onset of $5-\mathrm{HT}$.

$$
\frac{d\left[\text { Rafo }^{\circ}\right]}{d t}=\left(k_{\text {basal,Raf }}+k_{f, \text { Raf }}([5-H T]\rangle_{\tau_{\text {delay }}}\right)[R a f]-k_{b, \text { Raf }}\left[R^{2} f^{p}\right]
$$

$$
[R a f]=[R a f]_{\text {total }}-\left[R a f^{p}\right]
$$




$$
\frac{d[M E K 1]}{d t}=\frac{k_{b, M E K}\left[M E K 1^{p}\right]}{\left[M E K 1^{p}\right]+K_{M E K, 2}}-\frac{k_{f, M E K}\left[R a f^{p}\right][M E K 1]}{[M E K 1]+K_{M E K, 1}}
$$

$$
\frac{d\left[M E K 1^{p D}\right]}{d t}=\frac{k_{f, M E K}\left[\operatorname{Raf}^{p}\right]\left[M E K 1^{p}\right]}{\left[M E K 1^{p}\right]+K_{M E K, 1}}-\frac{k_{b, M E K}\left[M E K 1^{p D}\right]}{\left[M E K 1^{p D}\right]+K_{M E K, 2}}
$$

$$
\begin{gathered}
{\left[M E K 1^{p}\right]=[M E K 1]_{t o t a l}-[M E K 1]-\left[M E K 1^{p p}\right](E q .5)} \\
\frac{d[E R K]}{d t}=\frac{k_{b, E R K}\left[E R K^{p}\right]}{\left[E R K^{p}\right]+K_{E R K, 2}}-\frac{k_{f, E R K}\left[M E K 1^{p p}\right][E R K]}{[E R K]+K_{E R K, 1}}
\end{gathered}
$$

$$
\frac{d\left[E R K^{p D}\right]}{d t}=\frac{k_{f, E R K}\left[M E K 1^{p D}\right]\left[E R K^{p}\right]}{\left[E R K^{p}\right]+K_{E R K, 1}}-\frac{k_{b, E R K}\left[E R K^{p D}\right]}{\left[E R K^{p D}\right]+K_{E R K, 2}}
$$

$$
\left[E R K^{p}\right]=[E R K]_{\text {total }}-[E R K]-\left[E R K^{p p}\right]
$$

We found that p-p38 MAPK inhibits ERK activation (see Results). The mechanism of this inhibition is not yet determined. Two plausible hypotheses are that activated p38 MAPK enhances MEK1 dephosphorylation, or enhances ERK dephosphorylation, in both cases indirectly via activation of an undetermined phosphatase. In the absence of discriminating data, we simulated the former mechanism. Thus, the dephosphorylation rate of MEK1, $k_{b, M E K}$, was assumed to be enhanced by p-p38 MAPK (Eqs. 9, 10). $E_{p 38}$ represents this inhibitory effect of p-p38 MAPK. The Heaviside step function heav $\left[\left[M E K 1^{p P}\right]-\right.$ $\left[M E K 1^{p p}\right]_{\text {basal }}$ is 0 if MEK 1 is equal to or lower than the basal level (i.e., $\left[M E K 1^{p p}\right]_{\text {basal }}$ ) and is 1 if MEK1 is larger than the basal level. This function is used to support the empirical finding that p-p38 MAPK has no effect on the basal level of MEK1/2 (see Results).

$$
\begin{gathered}
k_{b, M E K}=k_{b, M E K \_ \text {basal }}+k_{b, M E K \_p 38} E_{p 38} \\
\frac{d\left[E_{p 38}\right]}{d t}=k_{E P 38}\left[P 38^{p D}\right] \operatorname{heav}\left(\left[M E K 1^{p D}\right]-\left[M E K 1^{p D}\right]_{b a s a l}\right) \\
-k_{d, E P 38} E_{p 38}
\end{gathered}
$$

\section{p38 MAPK pathway}

The mechanism underlying the inhibition and activation of p38 MAPK by 5-HT has yet to be characterized. Based on the empirical results of this study, we assumed that $5-\mathrm{HT}$ engages two separate pathways to, respectively, inhibit and activate p38 MAPK. In the first pathway, 5-HT inhibits the phosphorylation rate $\left(k_{f, p 38}\right)$ of p38 MAPK (Eqs. 19, 20). In the second pathway, a delayed phosphorylation of p38 MAPK due to 5-HT occurs via a p38 MAPKspecific-MAPKK kinase (MAPKKK)/MAPKK cascade (Eqs. 11-18) similar to the one that phosphorylates ERK; however, it is still unknown which isoform(s) of MAPKK phosphorylate p38 MAPK in Aplysia. [MAPKK ${ }_{p 38}$ ] represents this unknown MAPKK isoform. The differential equations describing the p38 MAPK pathway are,

$$
\begin{aligned}
& \frac{d\left[M A P K K K_{p 38}^{p}\right]}{d t}=\left(k_{\text {basal,Rafp } 38}+k_{f, \text { Rafp } 38}[5-H T]\right) \\
& \quad \times\left[M A P K K K_{p 38}\right]-k_{b, \text { Rafp } 38}\left[M A P K K K_{p 38}^{p}\right]
\end{aligned}
$$

$$
\left[M A P K K K_{p 38}\right]=\left[M A P K K K_{p 38}\right]_{\text {total }}-\left[M A P K K K_{p 38}^{p}\right]
$$

$$
\begin{gathered}
\frac{d\left[M A P K K_{p 38}\right]}{d t}=\frac{k_{b, M A P K K}\left[M A P K K_{p 38}^{p}\right]}{\left[M A P K K_{p 38}^{p}\right]+K_{M A P K K, 2}} \\
-\frac{k_{f, M A P K K}\left[M A P K K K_{p 38}^{p}\right]\left[M A P K K_{p 38}\right]}{\left[M A P K K_{p 38}\right]+K_{M A P K K, 1}} \\
\frac{d\left[M A P K K_{p 38}^{p p}\right]}{d t}=\frac{k_{f, M A P K K}\left[M A P K K K_{p 38}^{p}\right]\left[M A P K K_{p 38}^{p}\right]}{\left[M A P K K_{p 38}^{p}\right]+K_{M A P K K, 1}} \\
-\frac{k_{b, M A P K K}\left[M A P K K_{p 38}^{p p}\right]}{\left[M A P K K_{p 38}^{p D}\right]+K_{M A P K K, 2}}
\end{gathered}
$$

$$
\left[M A P K K_{p 38}^{p}\right]=\left[M A P K K_{p 38}\right]_{t o t a l}-\left[M A P K K_{p 38}\right]
$$$$
-\left[M A P K K_{p 38}^{p p}\right]
$$

$$
\frac{d[P 38]}{d t}=\frac{k_{b, p 38}\left[P 38^{p}\right]}{\left[P 38^{p}\right]+K_{p 38,2}}-\frac{k_{f, p 38}\left[M A P K K_{p 38}^{p p}\right][P 38]}{[P 38]+K_{p 38,1}}
$$

$$
\frac{d\left[p 38^{p p}\right]}{d t}=\frac{k_{f, p 38}\left[M A P K K_{p 38}^{p p}\right]\left[p 38^{p}\right]}{\left[p 38^{p}\right]+K_{p 38,1}}-\frac{k_{b, p 38}\left[p 38^{p p}\right]}{\left[p 38^{p p}\right]+K_{p 38,2}}
$$

$$
\begin{gathered}
{\left[p 38^{p}\right]=[p 38]_{\text {total }}-[p 38]-\left[p 38^{p p}\right]} \\
k_{f, p 38}=\frac{k_{f, p 38 \_\max }}{1+E_{5-H T}}
\end{gathered}
$$

$$
\frac{d\left[E_{5-H T}\right]}{d t}=k_{E 5 H T} \frac{[5-H T]}{[5-H T]+K_{5 H T \_p 38}}-k_{d, E 5 H T} E_{5-H T}
$$

$\left[p 38^{p p}\right]$ in Eq. 17 corresponds to the p-p38 MAPK level measured by immunofluorescence. The parameters of the p38 MAPK cascade were adjusted so that a pulse of 5-min 5-HT elicited a transient decrease, followed by a delayed increase, of p-p38 MAPK (see Results).

The standard parameter values for the ERK and p38 MAPK pathway model are: $k_{\text {basal,Raf }}=0.006 \mathrm{~min}^{-1}, k_{f, \text { Raf }}=$ $0.0023 \mu \mathrm{M}^{-1} \mathrm{~min}^{-1}, k_{b, \text { Raf }}=0.017 \mathrm{~min}^{-1},[\text { Raf }]_{\text {total }}=0.5$ $\mu \mathrm{M}, k_{f, M E K}=0.41 \mathrm{~min}^{-1}, k_{b, M E K \_ \text {basal }}=0.04 \mu \mathrm{M} / \mathrm{min}$, $k_{b, M E K \_D 38}=0.04 \mathrm{~min}^{-1}, K_{M E K, 1}=0.20 \mu \mathrm{M}, K_{M E K, 2}=0.19$ $\mu \mathrm{M},[\mathrm{MEK} 1]_{\text {total }}=0.5 \mu \mathrm{M}, k_{f, E R K}=0.41 \mathrm{~min}^{-1}, k_{b, E R K}=$ $0.12 \mu \mathrm{M} / \mathrm{min}, K_{E R K, 1}=0.19 \mu \mathrm{M}, K_{E R K, 2}=0.21 \mu \mathrm{M}$, $[E R K]_{\text {total }}=0.5 \mu \mathrm{M}, k_{E P 38}=0.5^{\mu} \mathrm{M}^{-1} \mathrm{~min}^{-1}$, 

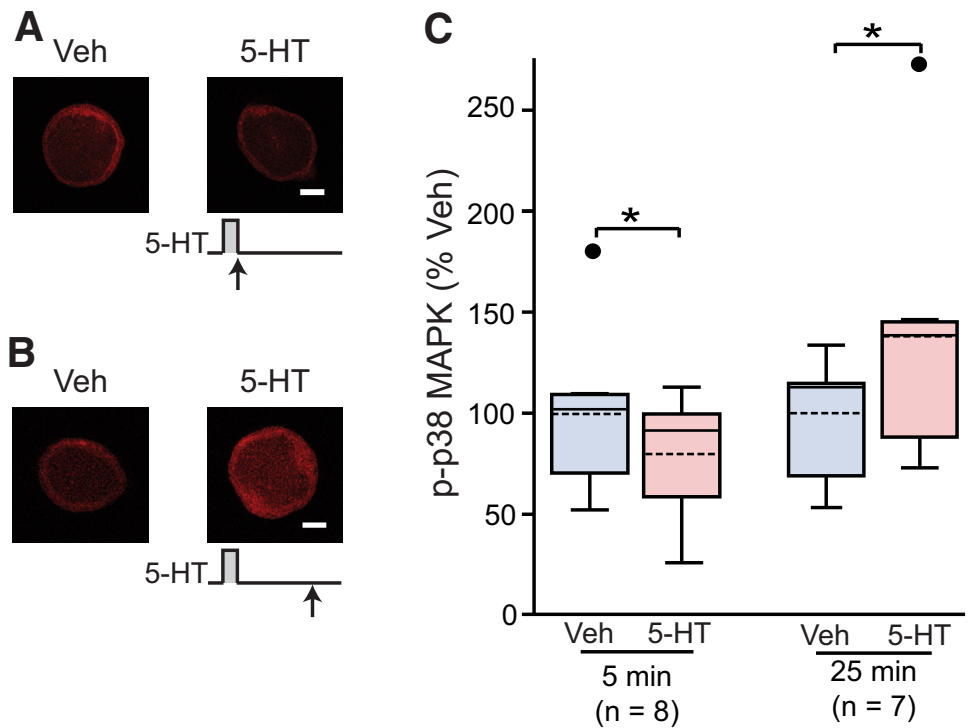

Figure 1. p-p38 MAPK at different times after one 5-min pulse of 5-HT (50 $\mu \mathrm{M})$. $\boldsymbol{A}$, Representative confocal images of p-p38 MAPK immunofluorescence in SNs immediately after the end of 5-HT. $\boldsymbol{B}$, Representative confocal images of p-p38 MAPK immunofluorescence in SNs at 25 min after onset of 5-HT. C, Summary data. p-p38 MAPK levels were normalized to the mean of Veh controls. Treatment with 5-HT for 5 min significantly decreased p-p38 MAPK immediately after the end of treatment, whereas the same treatment led a significant increase in p-p38 MAPK when measured 25 min after onset of $5-\mathrm{HT}$. In this and other illustrations, data are presented by box-and-whisker plots. The median is indicated by the solid line in the interior of the box. The mean is indicated by the dashed line in the interior of the box. The lower end of the box is the first quartile (Q1). The upper end of the box is the third quartile (Q3). The ends of the vertical lines (whiskers) are the maximum and minimum values of nonoutliers. The small circles outside the whiskers are the outliers larger than Q3 + 1.5(Q3-Q1) or smaller than Q3-1.5(Q3-Q1). Scale bar, $20 \mu \mathrm{m}$. Significant differences are indicated by $*$ for $p<0.05$.

$\left[M E K 1^{p p}\right]_{\text {basal }}=0.26 \mu \mathrm{M}, k_{d, E P 38}=0.1 \min ^{-1}, k_{\text {basal,Rafp38 }}$ $=0.029 \mathrm{~min}^{-1}, k_{f, \text { Rafp38 }}=0.003 \mu \mathrm{M}^{-1} \min ^{-1}, k_{b, \text { Rafp38 }}=$ $0.08 \mathrm{~min}^{-1},\left[\text { Raf }_{p 38}\right]_{\text {total }}=0.5 \mu \mathrm{M}, k_{f, M A P K K}=0.41 \mathrm{~min}^{-1}$, $k_{b, M A P K K}=0.04 \mu \mathrm{M} / \mathrm{min}, K_{\text {MAPKK,1 }}=0.20 \mu \mathrm{M}, K_{\text {MAPKK,2 }}$ $=0.19 \mu \mathrm{M},\left[\mathrm{MAPKK} K_{p 38}\right]_{\text {total }}=0.5 \mu \mathrm{M}, k_{f, p 38 \_ \text {max }}=0.41$ $\min ^{-1}, k_{b, p 38}=0.12 \mu \mathrm{M} / \mathrm{min}, K_{p 38,1}=0.19 \mu \mathrm{M}, K_{p 38,2}=$ $0.21 \mu \mathrm{M},[\mathrm{p} 38]_{\text {total }}=0.5 \mu \mathrm{M}, k_{E 5 H T}=0.25 \mathrm{~min}^{-1}, K_{5 H T \_p 38}$ $=50 \mu \mathrm{M}, k_{d, E 5 H T}=0.1 \mathrm{~min}^{-1}$.

\section{Numerical methods}

Fourth-order Runge--Kutta integration was used for integration of differential equations with a time step of $3 \mathrm{~s}$. No significant improvement in accuracy was found upon further time step reduction. Prior to any stimuli, the steady-state levels of variables were determined after at least two simulated days. The model was programmed in XPPAUT (RRID: SCR001996) and simulated on Dell precision T1700 computers. Source codes will be submitted to ModelDB (McDougal et al., 2015).

\section{Results}

\section{5-HT induced an initial decrease in p-p38 MAPK followed by an increase}

MAPK isoforms are activated by phosphorylation of the threonine and tyrosine residues in their activation loops. Thus, in this study, the levels of pERK and of p-p38 MAPK are used as measures of ERK and p38 MAPK activity. A 10 -min treatment of $50 \mu \mathrm{M} \mathrm{5-HT}$ to Aplysia in vivo produces a $\sim 30 \%$ reduction in p-p38 MAPK in Aplysia pleural ganglia as measured with Western blot (Guan et al.,
2003). However, it is not known whether a similar decrease is produced in individual SNs, nor have the dynamics of p-p38 MAPK at later time points been examined. We used immunofluorescence to quantify p-p38 MAPK in isolated SNs immediately or at a later time after treatment. Two treatment groups were examined: (1) one pulse of $5 \mathrm{~min} 5-\mathrm{HT}$, with fixation immediately after treatment ended; and (2) one pulse of $5 \mathrm{~min} 5-\mathrm{HT}$, fixed at 25 min after onset of 5-HT. Example responses are illustrated in Figure $1 A, B$, and summary data are in Figure $1 C$. 5 -HT led to a $20 \pm 6.2 \%(n=8 ; n$ here and also in other experiments represents the number of dishes) decrease in p-p38 MAPK levels measured immediately after treatment (WSRT, $Z=2.521 ; p=0.008)^{\text {a }}$ (superscript letters a-i refer to entries in the statistical table, see Table 1). These results indicate the $5-\mathrm{HT}$-induced decrease in p-p38 MAPK observed in ganglia by Guan et al. (2003) are also observed in individual SNs. We also found that the initial decrease was followed by a $39 \pm 17 \%(n=7)$ increase in p-p38 MAPK above control, at 25 min after the 5-min treatment (WSRT, $Z=2.197 ; p=0.03)^{\mathrm{b}}$.

To explore in more detail the time course of p38 MAPK after one pulse of $5-\mathrm{HT}$, immunofluorescence was used to quantify p-p38 MAPK in isolated SNs fixed at 5, 15, 45, and $60 \mathrm{~min}$ after onset of a $5-\mathrm{min}$ pulse of $50 \mu \mathrm{M} 5-\mathrm{HT}$. These time points and duration of 5-HT treatment correspond to those used to measure pERK (Philips et al., $2013 b)$. Figure $2 A, B$ illustrates that $5-\mathrm{HT}$ led to an initial reduction $(-8.6 \pm 7.2 \%, n=28)$ of p-p38 MAPK (the 5-min time point; see also Figure $1 C$ ). This initial decrease was 
Table 1: Statistical Table

\begin{tabular}{lllr}
\hline a & $\begin{array}{l}\text { Data structure } \\
\text { Non-normal distribution }\end{array}$ & $\begin{array}{l}\text { Type of test } \\
\text { Wilcoxon signed-rank test }\end{array}$ & $\begin{array}{c}\text { Power } \\
95 \% \text { confidence interval of } \\
\text { the difference: }-20.6 \text { to }-1.2\end{array}$ \\
b & Non-normal distribution & Wilcoxon signed-rank test & $\begin{array}{c}95 \% \text { confidence interval of } \\
\text { the difference: }-4.6 \text { to } 35.2\end{array}$ \\
& & & $95 \%$ confidence interval of \\
c & & Wilcoxon signed-rank test & difference: -16.7 to -0.4 \\
d & Non-normal distribution & & Power: 0.956 \\
e & Normal distribution & Paired $t$ test & Power: 0.992 \\
f & Normal distribution & Paired $t$ test & Power: 0.858 \\
g & Normal distribution & One-way RM ANOVA + Student--Newman--Keuls & Power: 0.805 \\
h & Normal distribution & One-way RM ANOVA + Student--Newman--Keuls & Power: 0.066 \\
i & Non-normal distribution & One-way ANOVA & Not applicable \\
& & Kruskal--Wallis one-way ANOVA on ranks &
\end{tabular}

followed by a return of p-p38 MAPK to near control level at $15 \mathrm{~min}(7.7 \pm 10.7 \%, n=14)$, an increase above control at $45 \min (26.7 \pm 6.8 \%, n=17)$, and then a return to control level at $60 \mathrm{~min}(4.7 \pm 7.6 \%, n=15)$. Statistical analyses revealed that the effects at the 5- and 45-min time points (Fig. 2C) were statistically significant (5 min, WSRT, $Z=2.960 ; p=0.003 ; 45 \mathrm{~min}$, paired $t$ test, $t_{(16)}=$ 3.669; $p=0.002)^{\mathrm{c}, \mathrm{d}}$. These results indicate that a brief pulse of 5-HT leads to a biphasic regulation of $\mathrm{p}-38$ MAPK, an initial decrease, followed by a late increase, and subsequent decline.

\section{p38 MAPK appears to mediate the decrease of pERK 60 min after $5-\mathrm{HT}$}

What is the functional significance of the late increase in p-p38 MAPK? Treatment with 5-HT increases the levels of pERK at $45 \mathrm{~min}$, which return to control level at $60 \mathrm{~min}$ (Philips et al., 2013b). The mechanism for this rapid drop is not known. One possibility is that the late increase in p-p38 MAPK (Fig. 2) produces a feed-forward late inhibition of MEK1/2 (Westermarck et al., 2001). If so, an inhibitor of p-p38 MAPK should boost pERK 60 min after treatment. The increase of $\mathrm{pERK}$ at 45 min was confirmed with immunofluorescence techniques in this study (data not shown). A 5-min treatment with $50 \mu \mathrm{M} 5$-HT led to a $48 \pm 11 \%$ increase in pERK $\left(n=8\right.$, paired $t$ test, $t_{(7)}=$ 5.112; $p<0.05)^{\mathrm{e}}$.

Next, immunofluorescence was used to examine levels of pERK at 60 min after onset of $5-\mathrm{HT}$, a time point where pERK is expected to have returned to baseline (Philips et al., 2013b). Measurements of pERK were made in the absence or presence of the p38 MAPK inhibitor SB203580. SB203580 was applied to SNs 30 min before and during $5 \mathrm{~min}, 50 \mu \mathrm{M}$ treatment with $5-\mathrm{HT}$. SNs continued to be treated with SB203580 until fixation at $60 \mathrm{~min}$ after onset of 5-HT. Four groups were examined: (1) 5-HT alone, (2) 3 $\mu \mathrm{M}$ SB203580 alone, (3) 5-HT + SB203580, and (4) Veh alone. Example responses are illustrated in Figure $3 A$, and summary data are presented in Figure $3 B$. Confirming Philips et al. (2013b), 5-HT alone had little effect ( $3 \pm$ $6.3 \%$ change) on pERK at $60 \mathrm{~min}(n=8)$. In contrast, 5 -HT led to a $36 \pm 11 \%(n=8)$ increase in pERK in the presence of SB203580.

A one-way repeated measures (RM) ANOVA revealed a significant overall effect of the treatments $\left(F_{(3,21)}=5.968\right.$, $p<0.01)^{f}$. Subsequent pairwise comparisons (Student-Newman--Keuls) revealed that the 5-HT + SB203580 group was significantly different from the Veh group ( $q=$ 5.217, $p<0.01, n=8)^{\mathrm{f}}$, from the 5-HT alone group $(q=$ 5.146, $p<0.01, n=8)^{f}$, and from the SB203580 alone group $(q=3.381, p=0.03, n=8)^{f}$. No significant difference was detected between the 5-HT alone group and the Veh group $(q=0.0717, p=0.96, n=8)^{f}$, between the SB203580 alone group and the Veh group $(q=1.837$, $p=0.41, n=8)^{\mathrm{f}}$, or between the $5-\mathrm{HT}$ alone group and the SB203580 alone group $(q=1.765, p=0.23, n=8)^{f}$. These results support the hypothesis that p38 MAPK is involved in the decrease of pERK at 60 min after 5-HT, possibly via inhibition of a MEK pathway.

\section{MAPKK is involved in the delayed phosphorylation of p38 MAPK by 5-HT}

The finding that the decrease of pERK 60 min after 5-HT results from an inhibitory effect of the p38 MAPK pathway raised a question whether the decrease of p38 MAPK 60 min after 5-HT results from a similar inhibitory effect of the ERK pathway. To explore the possibility of such mutual inhibition, immunofluorescence was used in pilot experiments (data not shown) to quantify p-p38 MAPK at 60 min after onset of $5-\mathrm{HT}$ in the presence of the MEK1/2 inhibitor U0126. U0126 was applied to SNs 30 min before and during 5-HT. SNs continued to be treated with U0126 until fixation, $60 \mathrm{~min}$ after onset of 5-min $50 \mu \mathrm{M} 5-\mathrm{HT}$. If the ERK pathway inhibits p38 MAPK activity, U0126 should relieve the inhibition and enhance p-p38 MAPK at $60 \mathrm{~min}$ after onset. Surprisingly, a small decrease of p-p38 MAPK by U0126 was observed. This result raised the intriguing possibility that U0126 may also attenuate the normal increase in p38 MAPK at 45 min after onset of 5-HT (Fig. 2).

To examine this possibility, U0126 was applied to SNs 30 min before and during 5-HT, and p-p38 MAPK was measured at $45 \mathrm{~min}$ after onset of $5-\mathrm{HT}$. SNs continued to be treated with U0126 until fixation, $45 \mathrm{~min}$ after onset of 5-min $50 \mu \mathrm{M} 5-\mathrm{HT}$. Four groups were examined: (1) 5-HT alone, (2) $20 \mu \mathrm{M}$ U0126 alone, (3) 5-HT + U0126, and (4) Veh alone. Example responses are illustrated in Figure $4 A 1$, and summary data are presented in Figure 4A2. Replicating the results of Figure 2, 5-HT led to an increase $(43 \pm 15 \%)$ in levels of p-p38 MAPK $(n=8)$. In contrast, 
A

$5 \min$

$15 \min$

$45 \mathrm{~min}$

$60 \mathrm{~min}$

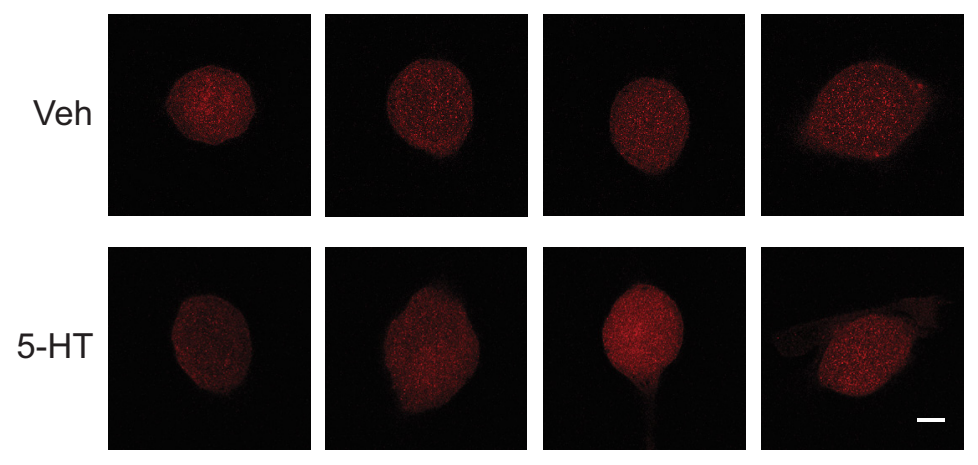

B

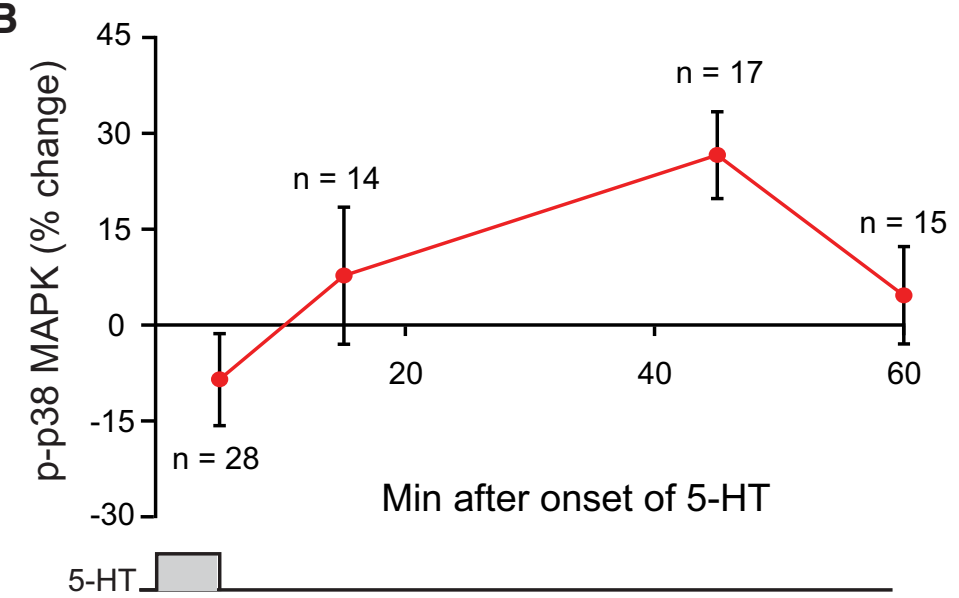

C

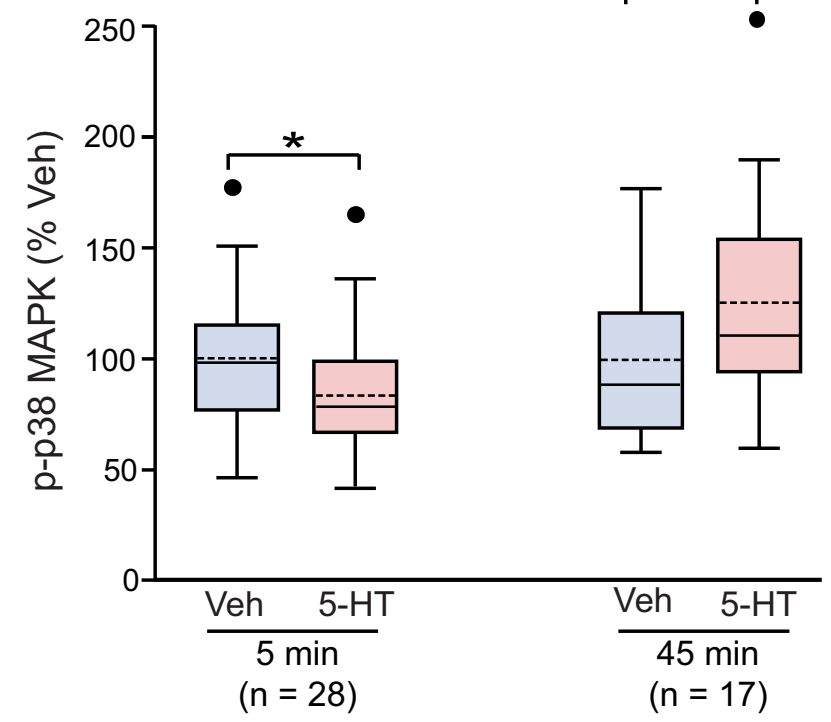

Figure 2. Biphasic regulation of p38 MAPK by one 5-min pulse of 5-HT (50 $\mu \mathrm{M})$. $\boldsymbol{A}$, Representative confocal images of p-p38 MAPK immunofluorescence in Aplysia SNs at different times after onset of 5-HT. Scale bar, $20 \mu \mathrm{m}$. B, Summary data. The percent change was calculated as the change of p-p38 MAPK level after 5-HT compared with control level. 5-HT induced a delayed increase in p-p38 MAPK, following a transient decrease immediately after treatment. p-p38 MAPK returned to the control level at 60 min. $\boldsymbol{C}$, Statistical analysis revealed significant differences between Veh and 5-HT treatment groups at 5 and 45 min. p-p38 MAPK levels were normalized to the mean of Veh controls. Significant differences between the groups are indicated by $*$ for $p<0.05$. 
A

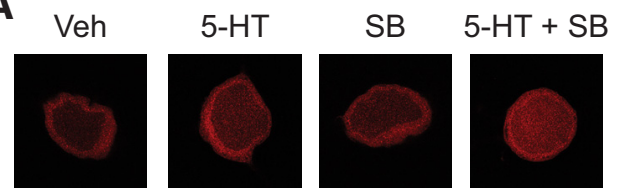

B

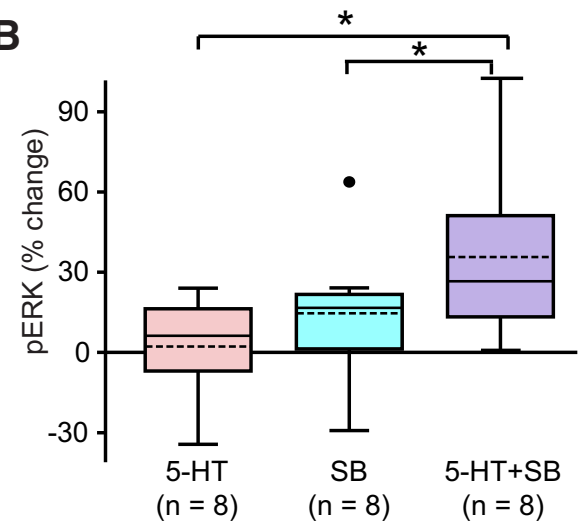

Figure 3. The effect of p38 MAPK inhibitor on pERK. $\boldsymbol{A}$, Representative confocal images of pERK immunofluorescence in SNs at $60 \mathrm{~min}$ after onset of a 5-min pulse of $5-\mathrm{HT}$, in the absence or presence of the p38 MAPK inhibitor SB203580 (SB). B, Summary data. Compared with the 5-HT alone group, treatment with 5-HT in the presence of SB induced a significant increase in pERK at $60 \mathrm{~min}$ after onset of $5-\mathrm{HT}$. Scale bar, $20 \mu \mathrm{m}$. Significant differences are indicated by $*$ for $p<0.05$

the increase was blocked $(2.1 \pm 11 \%)$ in the presence of U0126 $(n=8)$. A one-way RM ANOVA revealed a significant overall effect of the treatments $\left(F_{(3,21)}=5.383, p<\right.$ $0.01, n=8)^{\mathrm{g}}$. Subsequent pairwise comparisons (Student--Newman--Keuls) revealed that the 5-HT alone group was significantly different from the Veh group $(q=$ $4.045, p=0.01, n=8)^{9}$, from the $5-\mathrm{HT}+\mathrm{U} 0126$ group ( $q$ $=4.696, p<0.01, n=8)^{\mathrm{g}}$, and from the U0126 alone group $(q=4.98, p=0.01, n=8)^{\mathrm{g}}$. No significant difference was observed between the U0126 alone group and the Veh group $(q=0.935, p=0.79, n=8)^{9}$, between the $5-\mathrm{HT}+\mathrm{U} 0126$ group and the Veh group $(q=0.652, p=$ $0.65, n=8)^{\mathrm{g}}$, or between the $5-\mathrm{HT}+\mathrm{U} 0126$ group and the U0126 alone group $(q=0.284, p=0.84)^{g}$. These results indicate that U0126 can suppress p-p38 MAPK elevation at 45 min after $5-\mathrm{HT}$ onset, supporting the hypothesis that a p38 MAPK-specific-MAPKKK/MAPKK pathway is involved in the delayed phosphorylation of p38 MAPK by $5-\mathrm{HT}$. However, it is unclear which isoform of MAPKK is involved in the delayed phosphorylation of p38 MAPK in Aplysia. In mammalian cells, U0126 inhibits MEK1/2 but is not effective in inhibiting other MAPKK isoforms (e.g., MKK3 and MKK6) responsible for phosphorylation of p38 MAPK (Duncia et al., 1998). It is unclear whether U0126 is only effective in inhibiting MEK $1 / 2$ in Aplysia. It is possible that U0126 also inhibits a MAPKK isoform, distinct from MEK1/2, that activates p38 MAPK in Aplysia.

SB203580 inhibits downstream effects of p38 MAPK by preventing p-p38 MAPK from interacting with substrates (Cuenda and Rousseau, 2007). SB203580 was shown above to boost pERK at $60 \mathrm{~min}$, plausibly through releas-
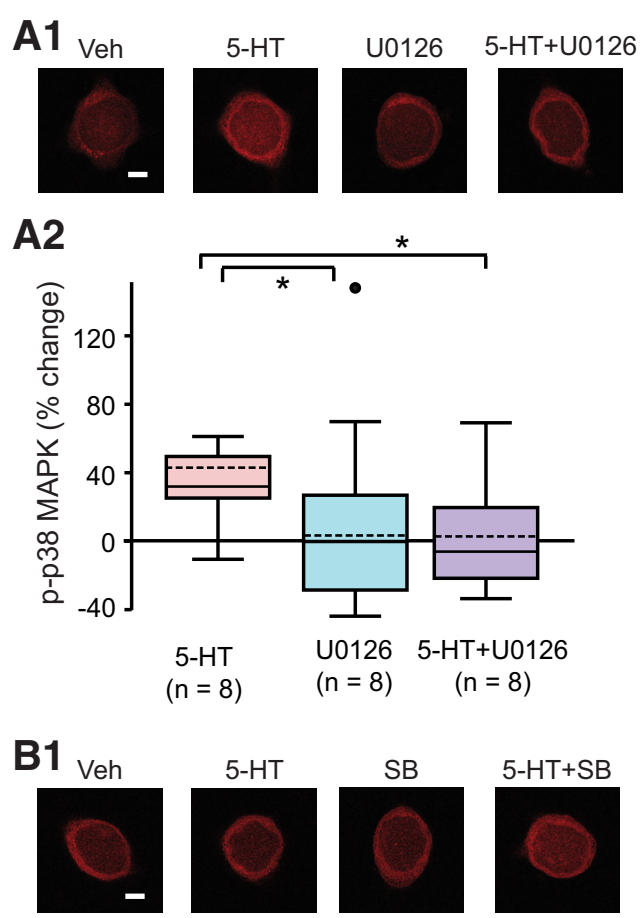

B2

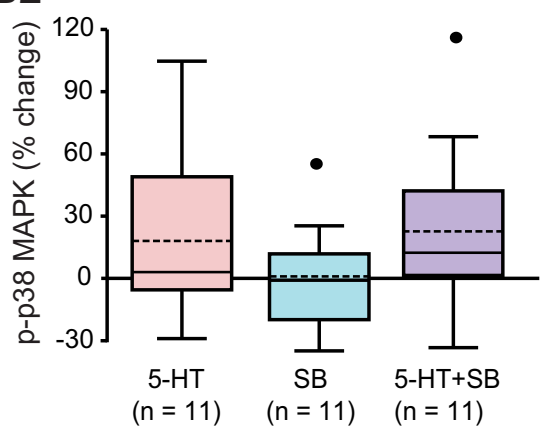

Figure 4. The interaction between p38 MAPK and MAPKK/ERK pathways. A1, Representative confocal images of p-p38 MAPK immunofluorescence in SNs at 45 min after onset of 5-HT, in the absence or presence of the MEK1/2 inhibitor U0126. A2, Summary data. Treatment with U0126 induced a significant decrease in 5-HT induced p-p38 MAPK at $45 \mathrm{~min}$. B1, Representative confocal images of p-p38 MAPK immunofluorescence in SNs at 60 min after onset of $5-\mathrm{HT}$, in the absence or presence of SB. B2, Summary data. No significant differences were found among the three groups. Scale bar, $20 \mu \mathrm{m}$. Significant differences are indicated by $*$ for $p<0.05$.

ing the suppression of MEK by p-p38 MAPK. If the same MEK isoforms, MEK1/2, do in fact activate p38 MAPK directly or indirectly via pERK, then p-p38 MAPK will form an autoinhibitory feedback loop, via suppression of MEK1. 5-HT alone had little effect on pERK at 60 min after onset, but a 5-HT-induced increase in pERK was observed in the presence of SB203580 (Fig. 3). Thus, SB203580 might be expected to also increase p-p38 MAPK at $60 \mathrm{~min}$ after onset of $5-\mathrm{HT}$ by breaking this inhibitory feedback loop. To investigate this hypothesis, immunofluorescence was used to quantify p-p38 MAPK in the presence of SB203580. SB203580 was applied to 
SNs 30 min before and during $5-\mathrm{HT}$. SNs continued to be treated with SB203580 until fixation, at $60 \mathrm{~min}$ after onset of 5-HT. Four groups were examined: (1) $50 \mu \mathrm{M} 5-\mathrm{HT}$ alone, (2) $3 \mu \mathrm{M}$ SB203580 alone, (3) 5-HT + SB203580, and (4) Veh alone. Example responses are illustrated in Figure $4 B 1$, and summary data are presented in Figure 4B2 . 5 -HT led to an $18 \pm 12 \%(n=11)$ change in p-p38 MAPK at 60 min after treatment, but a similar change (23 $\pm 12 \%, n=11$ ) was observed in the group that received the combination of 5-HT + SB203580. A one-way ANOVA found no significant overall effect of the treatments $\left(F_{(3,40)}\right.$ $=1.102, p=0.36)^{\mathrm{h}}$. Thus, SB203580 had no effect on 5 -HT-induced levels of p-p38 MAPK at 60 min after 5-HT (Fig. 4B), which does not support the possibility of an autoinhibitory loop for p38 MAPK. Combined with the findings that U0126 decreased pERK and p-p38 MAPK, the results indicate that $5-\mathrm{HT}$ may induce separate, but similar, MAPKK pathways to activate p38 MAPK and ERK.

\section{p38 MAPK phosphorylation at $\mathbf{4 5}$ min after onset of 5-HT treatment}

Philips et al. (2007; 2013b) found that two tail shocks to Aplysia could induce long-term sensitization of a withdrawal reflex but only when spaced by $45 \mathrm{~min}$. No LTM was observed with an ISI that was too short $(15 \mathrm{~min})$ or too long (60 min). Therefore, it is important to examine the dynamics of p-p38 MAPK in response to spaced and massed stimuli to obtain insights into whether the delayed activation of p-p38 MAPK by 5-HT is involved in determining the efficacy of different protocols to induce LTM.

Immunofluorescence of isolated $\mathrm{SNs}$ was used to quantify p-p38 MAPK after four different 5 -HT treatments: a 25-min continuous application of 5-HT (i.e., massed), two 5 -min applications separated by $20 \mathrm{~min}$ or $45 \mathrm{~min}$ (i.e., spaced), and a single 5-min application (control group). An ISI of 20 min is commonly used in 5-HT protocols to induce LTF (Martin et al., 1997; Sharma et al., 2003; Zhang et al., 2012). Because pERK increases $\sim 45$ min after the onset of 5-HT (Philips et al., 2013b and confirmed herein) and two stimuli separated by $45 \mathrm{~min}$ lead to LTM (Philips et al., 2007), it is important to quantify p-p38 MAPK 45 min after 5-HT onset and compare the effects of different ISIs (20 vs $45 \mathrm{~min}$ ). A stimulus protocol that significantly decreases p-p38 MAPK would plausibly be effective in induction of LTF. Four treatment groups were examined: (1) one pulse of $5 \mathrm{~min} 5-\mathrm{HT}$ and fixed at 45 min after onset of 5-HT; (2) two 5-min pulses with ISI of $20 \mathrm{~min}$ and fixed $20 \mathrm{~min}$ after 5-HT treatment ended; (3) one 25 min pulse as used by Mauelshagen et al. (1998) and fixed 20 min after treatment ended; and (4) two 5-min pulses with ISI of $45 \mathrm{~min}$ and fixed immediately after treatment ended. In the first three groups, p-p38 MAPK was measured at $45 \mathrm{~min}$ after $5-\mathrm{HT}$ onset, whereas p-p38 MAPK was measured 50 min after 5 -HT onset in the fourth group. Example responses are illustrated in Figure $5 A$, and summary data are presented in Figure $5 B ; 45 \mathrm{~min}$ after $5-\mathrm{HT}$ onset, two pulses with an ISI of 20 min led to a $27 \pm 7.6 \%$ increase in p-p38 MAPK $(n=9)$, whereas the increase from $25 \mathrm{~min} 5-\mathrm{HT}$ was $30 \pm 8.0 \%(n=9)$. In contrast, two pulses with an ISI of 45 min only increased p-p38 MAPK by $9 \pm 11 \%(n=9)$. Consistent with data of Figure 2, one pulse of 5-HT produced a substantial increase $(35 \pm 9.2 \%, n=9)$ in levels of p-p38 MAPK $45 \mathrm{~min}$ after onset.

Kruskal--Wallis one-way ANOVA on ranks revealed a significant overall effect of the treatments $\left(H_{3}=9.531, p\right.$ $<0.05)^{\mathrm{i}}$. Subsequent pairwise comparisons (Student-Newman--Keuls) revealed that the group receiving two pulses of 5-HT with ISI of 45 min was significantly different from the other groups (vs one-pulse group: $q=4.018, p$ $<0.05$; vs two pulses with ISI of 20 min: $q=4.368, p<$ 0.05 ; vs $25 \min 5-\mathrm{HT}: q=6.181, p<0.05)^{\mathrm{i}}$. No significant difference was detected among the other groups.

In SNs treated with two pulses of 5-HT with an ISI of 45 min, p-p38 MAPK remained around the control level $\sim 50$ min after onset of $5-\mathrm{HT}$. This low level of p-p38 MAPK immediately after the 45-min pulse might facilitate the induction of LTF. In contrast, the high level of p-p38 MAPK at $45 \mathrm{~min}$ after the massed protocol may contribute to a state that is not permissive for LTF.

\section{A computational model predicted roles of pERK and p-p38 MAPK in determining the efficacy of spaced 5-HT protocols to induce LTF}

To help provide insights into the possible consequences of the dynamics of p38 MAPK and ERK on the induction of LTF, a computational model was developed (Fig. $6 \mathrm{~A}$ and Materials and Methods). A key model assumption is that $5-\mathrm{HT}$ inhibits and activates p-p38 MAPK through two independent pathways. The inhibitory pathway acts rapidly and predominates during exposure to $5-\mathrm{HT}$. The stimulatory pathway is MEK dependent and activates slowly and becomes predominant after 5-HT washout. Model parameters were initially constrained so that a single 5-min pulse of 5-HT elicited a delayed phosphorylation of ERK (Fig. 6B1) as well as a transient inhibition, followed by a delayed phosphorylation of p38 MAPK (Fig. 6B2). The empirical data used to constrain the model parameters were: the levels of pERK at 45 and 60 min after onset of 5-HT (Fig. 6B1) and of p-p38 MAPK at $5,15,25,45$, and 60 min after onset of 5-HT (Fig. 6B2). These data were obtained from the experiments of Figures 1-5, except that pERK at 45 min was measured in additional experiments. The simulation results (black curves) are similar to the empirical data (red circles). The simulation of pERK dynamics (Fig. 6B1) is also consistent with the time course measurements by Philips et al. (2013b).

The model was then used to simulate the time courses of pERK and p-p38 MAPK after two pulses of 5-HT treatment with ISI of $45 \mathrm{~min}$ (Fig. 7A1). The simulations exhibited a phase shift between the peaks of pERK and p-p38 MAPK. Approximately 50 min after onset of $5-\mathrm{HT}$, when pERK remained elevated, p-p38 MAPK approached its control level (Fig. 7A1, black arrows), consistent with empirical results in Figure 5. To quantify the differential effects of two-pulse 5-HT protocols with different ISIs, the time course of the ratio of pERK to p-p38 MAPK was simulated for $2 \mathrm{~h}$ after onset of $5-\mathrm{HT}$ (Fig. 7A1, red curve), and compared with the time course of the ratio of $p E R K$ to 

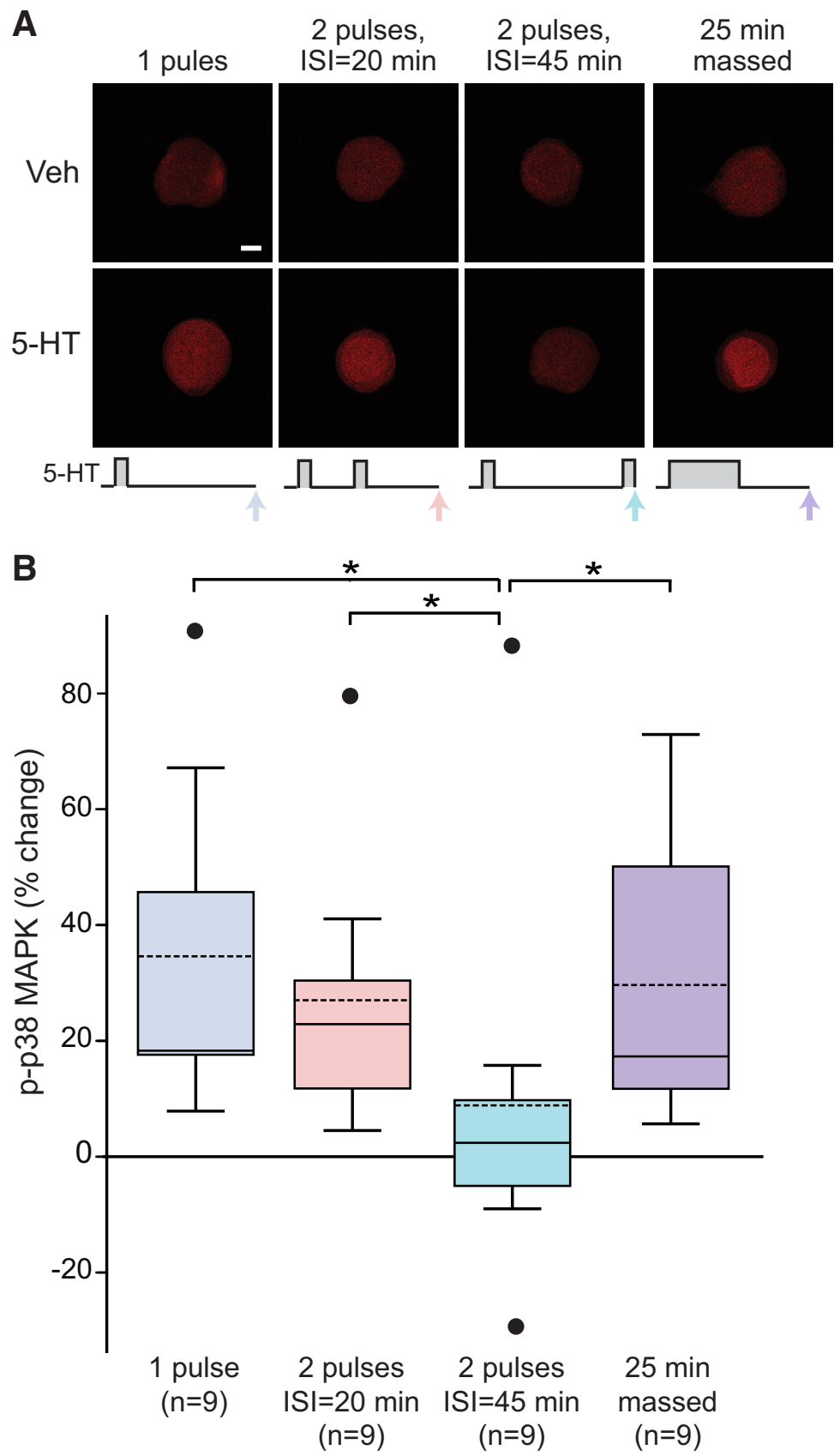

Figure 5. p-p38 MAPK 45-50 min after onset of different stimulus protocols. A, Representative confocal images of p-p38 MAPK immunofluorescence in SNs at 45-50 min after onset of 5-HT. B. Summary data. Levels of p-p38 MAPK was measured at 45 min after onset of a single 25-min 5-HT pulse, two 5-min duration pulses of 5-HT with ISI of 20 min, or one 5-min duration pulse of 5-HT. p-p38 MAPK was measured at 50 min after onset of two pulses of 5-HT with an ISI of 45 min. The last group produced less p-p38 MAPK than any of three former groups. Scale bar, $20 \mu \mathrm{m}$. Significant differences are indicated by $*$ for $p<0.05$.

p-p38 MAPK from one pulse (Fig. 7A1, black curve). The peak ratio of $p E R K$ to $p$-p38 MAPK after one pulse occurred immediately after the end of $5-\mathrm{HT}$, due to the decrease of p-p38 MAPK when pERK remained at basal level. The peak ratio of pERK to $p$-p38 MAPK after one pulse was $\sim 1.2$ (dashed line). The peak ratio of pERK to p-p38 MAPK after two pulses of 5-HT treatment with ISI of 45 min was $\sim 1.3$, occurring at about $50 \mathrm{~min}$. It is apparent that a second pulse with ISI of 45 min produced a greater peak ratio of pERK to $p-p 38$ MAPK than the first pulse. In contrast, a second pulse with ISI of $60 \mathrm{~min}$ failed to produce a greater peak ratio of $\mathrm{pERK}$ to $\mathrm{p}$-p38 MAPK than the first pulse (Fig. 7A2), and neither did a second pulse with ISI of 20 min (data not shown). Given that ERK phosphorylation is associated with LTF, whereas p38 MAPK phosphorylation is associated with LTD, these results suggest a possible explanation for why the ISI of 45 min is superior in inducing LTM: only with an ISI of $45 \mathrm{~min}$ 
A 1

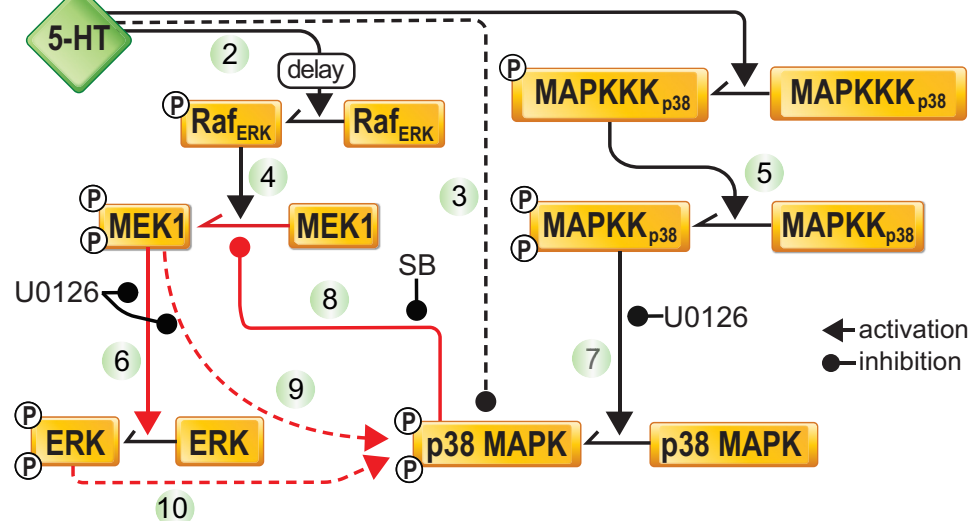

B1

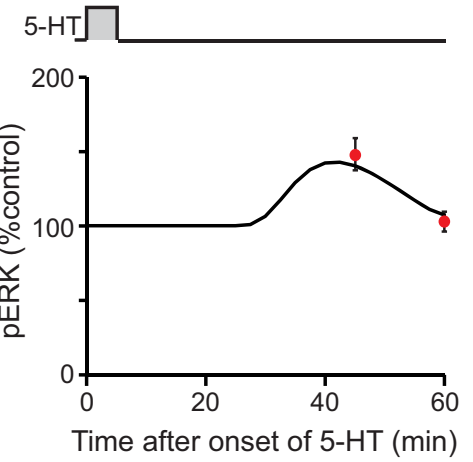

B2
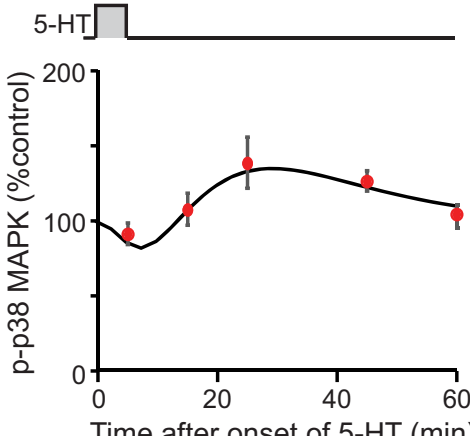

Figure 6. Computational simulation of ERK and p38 MAPK phosphorylation after different $5-\mathrm{HT}$ treatments. $\boldsymbol{A}$, Schematic of the model of the ERK and p38 MAPK signaling pathways. Key pathways are labelled by numbers. $\boldsymbol{B}$, Dynamics of pERK (B1) and p-p38 MAPK (B2) after one pulse of 5-HT. The simulations (black traces) are qualitatively similar to the empirical data (red circles) collected in the present study.

is the peak ratio of pERK to $p$-p38 MAPK higher than the peak produced by one pulse.

The model in Zhang et al. (2012), which lacked the p38 MAPK pathway, predicted, and empirically validated, a novel enhanced protocol of five 5-min pulses of 5-HT that produced stronger LTF and LTM than the standard protocol commonly used to induce LTF in Aplysia (five 5-min pulses of 5-HT, with uniform ISI of $20 \mathrm{~min}$ ) (Fig. 7B1). This enhanced protocol has irregular ISIs $(10,10,5,30 \mathrm{~min})$ (Fig. 7B2), which help generate a greater overlap of PKA and ERK activities than the standard protocol does. With the current model, the peak ratio of pERK to p-p38 MAPK after the enhanced protocol was $\sim 1.8$ (Fig. 7B2), whereas the peak ratio of $p E R K$ to $p-p 38$ MAPK after the standard protocol was less, $\sim 1.4$ (Fig. 7B1). This greater peak ratio may also contribute to the efficacy of the enhanced protocol.

\section{Discussion}

\section{Dynamic regulation of p38 MAPK activity}

Previously, Guan et al. (2003) hypothesized that the 5-HT-induced inhibition of p38 MAPK activity (Fig. 1, 2, $6 A$, pathway 3 ) relieved an inhibitory constraint on the induction of LTF. Results from the present study indicate that this previous hypothesis needs to be extended. Our results indicate that $5-\mathrm{HT}$ also elicited a late increase in p38 MAPK activity. This biphasic regulation of p38 MAPK by $5-\mathrm{HT}$ is likely to have profound consequences for the understanding of the induction of LTF at sensorimotor synapses of Aplysia and possibly other forms of long-term plasticity in other systems. For example, spaced learning is, in general, superior to massed learning in formation of LTM or LTF (Donovan and Radosevich, 1999; Cepeda et al., 2006; Vlach, 2014; Smolen et al., 2016), but not all spaced learning protocols are efficient in memory formation. Philips et al. (2007) found that in Aplysia, two tail shocks with ISI of $45 \mathrm{~min}$ form LTM, but shorter (15 min) or longer (60 $\mathrm{min}$ ) ISIs fail to induce LTM. Zhang et al. (2012) used a computational model to suggest that the amount of overlap between PKA and ERK pathways plays a critical role in determining the efficacy of spaced protocols. Overlap between PKA and ERK activities was greatest when the two-pulse protocol was simulated with ISI of $45 \mathrm{~min}$. Here, we extended this model to include the regulation of p38 MAPK by $5-\mathrm{HT}$. Data suggest that the dynamics of p38 MAPK and ERK are also important for determining the efficacy of spaced learning, leading us to hypothesize that the ratio of ERK to p38 MAPK activity may contribute to the efficacy of spaced 5-HT protocols. In particular, data indicate that two pulses of 5-HT with an 
A1
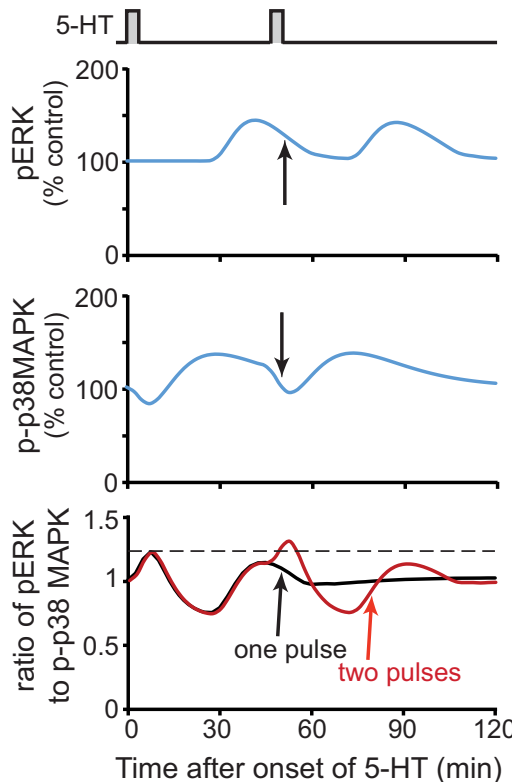

B1

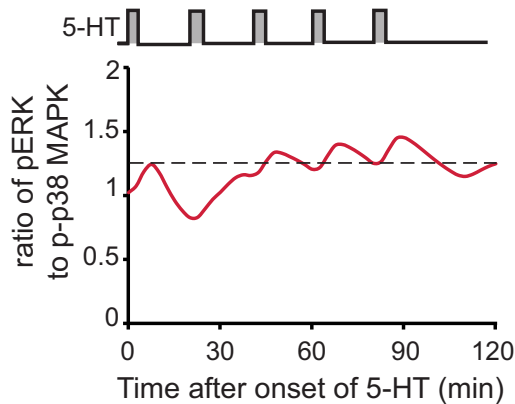

A2
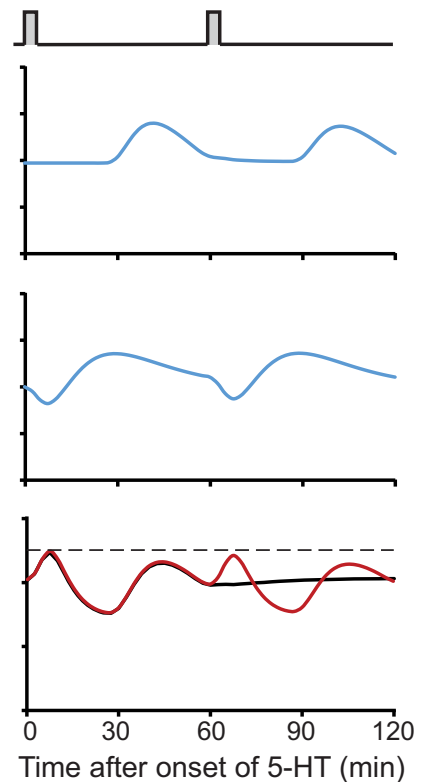

\section{B2}

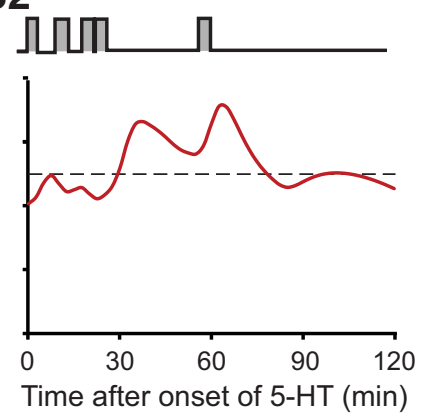

Figure 7. Computational simulation of the ratio of pERK to p-p38 MAPK after two or five pulses of $5-\mathrm{HT}$. $\boldsymbol{A}$, Dynamics of pERK and p-p38 MAPK levels, and the ratio of pERK to p-p38 MAPK, for 2 hours after two pulses with ISI of 45 min (A1) or 60 min (A2). $\boldsymbol{B}$, Dynamics of the ratio of pERK to p-p38 MAPK for two hours after five pulses with ISIs of the standard protocol (B1) or enhanced protocol (B2) as compared with the peak ratio of pERK to p-p38 MAPK after one pulse ( 1.2, dashed line).

ISI of 45 min, but not 20 or 60 min, induce LTM. Corresponding simulations indicate that an ISI of $45 \mathrm{~min}$ increased the ratio of $\mathrm{pERK}$ to $\mathrm{p}-\mathrm{p} 38$ MAPK more than did the protocols with ISI of $20 \mathrm{~min}$ or $60 \mathrm{~min}$, or one pulse of 5-HT (Fig. 7A). Thus, the 45-min ISI appears to leverage the dynamics of the PKA, ERK, and p38 MAPK pathways to produce superior long-term plasticity. In mammals, p38 MAPK activity is also important for LTD (Bolshakov et al., 2000; Murray and O'Connor, 2003). However, the effects of p38 MAPK in Aplysia and mammals differ in that p38 MAPK inhibition does not affect LTP in the rat dentate gyrus (Murray and O'Connor, 2003), whereas it enhances LTF in Aplysia (Guan et al., 2003).

\section{Cross talk between ERK and p38 MAPK pathways}

5-HT leads to a delayed activation of ERK, which peaks at about 45 min (Philips et al., 2007). Interestingly, the increase rapidly declines by 60 min. SB203580 inhibits downstream effects of p38 MAPK by binding the ATPbinding pocket of p38 MAPK, therefore preventing p38
MAPK from interacting with substrates (Cuenda and Rousseau, 2007). It was found that SB203580 increased pERK at 60 min after onset of 5-HT, suggesting that p38 MAPK inhibits ERK activation (Fig. 3), possibly through inhibition of MEK (Westermarck et al., 2001; Fig. 6A, pathway 8). Indeed, studies in human embryonic fibroblasts suggest that p38 MAPK inhibits the MAPK kinase (MAPKK) isoforms MEK1/2, the upstream activators of ERK (Shaul and Seger, 2007), by enhancing their dephosphorylation (Westermarck et al., 2001; Junttila et al., 2008). Thus, it is possible that p38 MAPK is responsible for the reduction of ERK activity 60 min after 5-HT. A delay evidently exists between the activation of p38 MAPK, 45 min after 5-HT onset, and the subsequent decline of ERK, $\sim 60$ min after 5-HT onset. This delay suggests that indirect inhibition of ERK activation by p38 MAPK includes one or more relatively slow intermediate molecular steps.

Such inhibition of ERK by p38 MAPK might help to explain the observations of some previous studies. For 
example, Guan et al. (2002) found that if FMRFa and 5-HT are applied to neurons simultaneously, LTD prevails over LTF. It is possible that the activation of p38 MAPK by FMRFa inhibits ERK, accounting in part for the override of LTF by LTD. Liu et al. (2014) found that treatment with a cancer chemotherapeutic drug doxorubicin (DOX) increases pERK and p-p38 MAPK at the same time in Aplysia SNs, but DOX suppresses 5-HT-induced LTF and enhances FMRFa-induced LTD. These results may be, at least partially, due to inhibition of the ERK pathway by p38 MAPK. In the present study, SB203580 did not change the level of p-p38 MAPK itself (Fig. 4B), suggesting that p38 MAPK does not induce a self-inhibitory feedback loop after its delayed phosphorylation (Fig. 6A, pathways $9->8->9$ or pathways $6->10->8->6)$. We note that our current model and simulations (Figs. 6,7 ) do not include other MAPKK or MAPK isoforms (e.g., JNK MAPK isoforms), the activation dynamics of which might influence pERK or p38 MAPK activities. Extant data do not, however, appear to substantially constrain and, thus, allow simulation of these possible modes of cross talk.

The mechanism underlying the delayed increase in p38 MAPK activity (Fig. 2) is unknown. It may be protein synthesis-dependent, similar to the delayed ( $\sim 45 \mathrm{~min}$ ) increase in ERK activity (Philips et al., 2013b). However, protein synthesis dependence cannot be examined with the commonly used protein synthesis inhibitors emetine and anisomycin, because emetine and anisomycin have been reported to activate p38 MAPK (Barros et al., 1997; Kim et al., 2015).

It would also be of interest to examine whether at these later times after 5-HT ( $\sim 45 \mathrm{~min})$, CREB2 is phosphorylated by p38 MAPK, as was observed by Guan et al. (2002, 2003) after FMRFa. A delayed phosphorylation of CREB2 might, by displacing CREB1 from the promoter of genes regulated by CREs, limit the amount of transcription underlying the induction of LTF, thus contributing to a "ceiling" on the amplitude of LTF.

Interestingly, the increase in p38 MAPK activity appears dependent on a MAPKK pathway, because it is attenuated by application of U0126 (Fig. 4A). However, the details of this pathway remain unclear and need to be investigated in future work. For example, U0126 is considered a highly selective inhibitor of MEK $1 / 2$ in mammalian cells and not effective in inhibiting two other MAPKKs, MKK3/6 (Duncia et al., 1998). U0126 suppresses the activity of ERK in Aplysia (Sharma et al., 2003). In the present study, U0126 also inhibited the activation of p38 MAPK in Aplysia (Fig. 4A). This result indicates that if U0126 also selectively inhibits a MEK1/2 analogue in Aplysia, p38 MAPK should be activated by MEK1/2 analogue in Aplysia, not by an MKK3/6 analogue as observed in mammalian cells (Raingeaud et al., 1996; Shaul and Seger, 2007). Alternatively, the action of U0126 may be less selective in Aplysia, inhibiting a MAPKK isoform such as MKK3/6 and, thereby, decreasing p38 MAPK activity. Further investigation is needed to determine whether U0126 can inhibit MKK3 and MKK6 analogues in Aplysia SNs, as well as whether such pathways activate p38 MAPK in Aplysia.

\section{References}

Adams JP, Sweatt JD (2002) Molecular psychology: roles for the ERK MAP kinase cascade in memory. Annu Rev Pharmacol Toxicol 42:135-163. CrossRef Medline

Ajay SM, Bhalla US (2004) A role for ERKII in synaptic pattern selectivity on the time-scale of minutes. Eur J Neurosci 20:26712680. CrossRef Medline

Barros LF, Young M, Saklatvala J, Baldwin SA (1997) Evidence of two mechanisms for the activation of the glucose transporter GLUT1 by anisomycin: p38 (MAP kinase) activation and protein synthesis inhibition in mammalian cells. J Physiol 504:517-525. CrossRef

Bartsch D, Ghirardi M, Skehel PA, Karl KA, Herder SP, Chen M, Bailey CH, Kandel ER (1995) Aplysia CREB2 represses long-term facilitation: relief of repression converts transient facilitation into long-term functional and structural change. Cell 83:979-992. Medline

Bolshakov VY, Carboni L, Cobb MH, Siegelbaum SA, Belardetti F (2000) Dual MAP kinase pathways mediate opposing forms of long-term plasticity at CA3-CA1 synapses. Nat Neurosci 3:11071112. CrossRef Medline

Cepeda NJ, Pashler H, Vul E, Wixted JT, Rohrer D (2006) Distributed practice in verbal recall tasks: a review and quantitative synthesis. Psychol Bull 132:354-380. CrossRef Medline

Choi YH, Lee SN, Aoyagi H, Yamasaki Y, Yoo JY, Park B, Shin DM, Yoon HG, Yoon JH (2011) The extracellular signal-regulated kinase mitogen-activated protein kinase/ribosomal S6 protein kinase 1 cascade phosphorylates cAMP response element-binding protein to induce MUC5B gene expression via D-prostanoid receptor signaling. J Biol Chem 286:34199-34214. CrossRef Medline

Cuenda A, Rousseau S (2007) p38 MAP-kinases pathway regulation, function and role in human diseases. Biochim Biophys Acta 1773: 1358-1375. CrossRef Medline

Donovan JJ, Radosevich DJ (1999) A meta-analytic review of the distribution of practice effect. J Appl Psychol 84:795-805. CrossRef

Duncia JV, Santella JB 3rd, Higley CA, Pitts WJ, Wityak J, Frietze WE, Rankin FW, Sun JH, Earl RA, Tabaka AC, Teleha CA, Blom KF, Favata MF, Manos EJ, Daulerio AJ, Stradley DA, Horiuchi K, Copeland RA, Scherle PA, Trzaskos JM, et al. (1998) MEK inhibitors: the chemistry and biological activity of U0126, its analogs, and cyclization products. Bioorg Med Chem Lett 8:2839-2844. Medline

English JD, Sweatt JD (1997) A requirement for the mitogenactivated protein kinase cascade in hippocampal long term potentiation. J Biol Chem 272:19103-19106. Medline

Fioravante D, Smolen PD, Byrne JH (2006) The 5-HT- and FMRFaactivated signaling pathways interact at the level of the Erk MAPK cascade: potential inhibitory constraints on memory formation. Neurosci Let 396:235-240. CrossRef

Guan Z, Giustetto M, Lomvardas S, Kim JH, Miniaci MC, Schwartz $\mathrm{JH}$, Thanos D, Kandel ER (2002) Integration of long-term-memoryrelated synaptic plasticity involves bidirectional regulation of gene expression and chromatin structure. Cell 111:483-493. Medline

Guan Z, Kim JH, Lomvardas S, Holick K, Xu S, Kandel ER, Schwartz JH (2003) p38 MAP kinase mediates both short-term and longterm synaptic depression in Aplysia. J Neurosci 23:7317-7325. Medline

Hu JY, Glickman L, Wu F, Schacher S (2004) Serotonin regulates the secretion and autocrine action of a neuropeptide to activate MAPK required for long-term facilitation in Aplysia. Neuron 43:373-385. CrossRef Medline

Junttila VR, Li SP, Westermarck J (2008) Phosphatase-mediated crosstalk between MAPK signaling pathways in the regulation of cell survival. FASEB J 22:954-965. CrossRef

Kim JH, Cho EB, Lee J, Jung O, Ryu BJ, Kim SH, Cho JY, Ryou C, Lee SY (2015) Emetine inhibits migration and invasion of human non-small-cell lung cancer cells via regulation of ERK and p38 
signaling pathways. Chem Biol Interact 242:25-33. CrossRef Medline

Liu R-Y, Zhang Y, Coughlin B, Cleary LJ, Byrne JH (2014) Doxorubicin attenuates serotonin-induced long-term synaptic facilitation by phosphorylation of p38 mitogen-activated protein kinase. $J$ Neurosci 34:13289-13300. CrossRef Medline

Martin KC, Michael D, Rose JC, Barad M, Casadio A, Zhu H, Kandel ER (1997) MAP kinase translocates into the nucleus of the presynaptic cell and is required for long-term facilitation in Aplysia. Neuron 18:899-912. Medline

Mauelshagen J, Sherff CM, Carew TJ (1998) Differential induction of long-term synaptic facilitation by spaced and massed applications of serotonin at sensory neuron synapses of Aplysia californica. Learn Mem 5:246-256. Medline

McDougal RA, Morse TM, Hines ML, Shepherd GM (2015) ModelView for ModelDB: online presentation of model structure. Neuroinformatics 13:459-470. CrossRef Medline

Michael D, Martin KC, Seger R, Ning MM, Baston R, Kandel ER (1998) Repeated pulses of serotonin required for long-term facilitation activate mitogen-activated protein kinase in sensory neurons of Aplysia. Proc Natl Acad Sci USA 95:1864-1869. Medline

Montarolo PG, Kandel ER, Schacher S (1988) Long-term heterosynaptic inhibition in Aplysia. Nature 333:171-174. CrossRef Medline

Murray HJ, O'Connor JJ (2003) A role for COX-2 and p38 mitogen activated protein kinase in long-term depression in the rat dentate gyrus in vitro. Neuropharmacology 44:374-380. Medline

Ormond J, Hislop J, Zhao Y, Webb N, Vaillaincourt F, Dyer JR, Ferraro G, Barker P, Martin KC, Sossin WS (2004) ApTrkl, a Trk-like receptor, mediates serotonin- dependent ERK activation and longterm facilitation in Aplysia sensory neurons. Neuron 44:715-728. CrossRef Medline

Philips GT, Tzvetkova El, Carew TJ (2007) Transient mitogenactivated protein kinase activation is confined to a narrow temporal window required for the induction of two-trial long-term memory in Aplysia. J Neurosci 27:13701-13705. CrossRef Medline

Philips GT, Kopec AM, Carew TJ (2013a) Pattern and predictability in memory formation: from molecular mechanisms to clinical relevance. Neurobiol Learn Mem 105:117-124.

Philips GT, Ye X, Kopec AM, Carew TJ (2013b) MAPK establishes a molecular context that defines effective training patterns for longterm memory formation. J Neurosci 33:7565-7573.

Purcell AL, Sharma SK, Bagnall MW, Sutton MA, Carew TJ (2003) Activation of a tyrosine kinase-MAPK cascade enhances the in- duction of long-term synaptic facilitation and long-term memory in Aplysia. Neuron 37:473-484. Medline

Raingeaud J, Whitmarsh AJ, Barrett T, Dérijard B, B, Davis RJ (1996) MKK3- and MKK6-regulated gene expression is mediated by the p38 mitogen-activated protein kinase signal transduction pathway. Mol Cell Biol 16:1247-1255. Medline

Schacher S, Montarolo P, Kandel ER (1990) Selective short- and long-term effects of serotonin, small cardioactive peptide, and tetanic stimulation on sensorimotor synapses of Aplysia in culture. J Neurosci 10:3286-3294.

Schacher S, Wu F, Sun ZY, Wang D (2000) Cell-specific changes in expression of mRNAs encoding splice variants of Aplysia cell adhesion molecule accompany long-term synaptic plasticity. J Neurobiol 15:152-161. CrossRef

Sharma SK, Carew TJ (2004) The roles of MAPK cascades in synaptic plasticity and memory in Aplysia: facilitatory effects and inhibitory constraints. Learn Mem 11:373-378. CrossRef Medline

Sharma SK, Sherff CM, Shobe J, Bagnall MW, Sutton MA, Carew TJ (2003) Differential role of mitogen-activated protein kinase in three distinct phases of memory for sensitization in Aplysia. J Neurosci 23:3899-3907.

Shaul YD, Seger R (2007) The MEK/ERK cascade: from signaling specificity to diverse functions. Biochim Biophys Acta 1773:12131226. 121CrossRef Medline

Smolen P, Zhang Y, Byrne JH (2016) The right time to learn: mechanisms and optimization of spaced learning. Nat Rev Neurosci 17:77-88. CrossRef Medline

Vlach HA (2014) The spacing effect in children's generalization of knowledge: allowing children time to forget promotes their ability to learn. Child Devel Persp 8:163-168. CrossRef

Westermarck J, Li SP, Kallunki T, Han J, Kähäri VM (2001) p38 mitogen-activated protein kinase-dependent activation of protein phosphatases 1 and $2 \mathrm{~A}$ inhibits MEK1 and MEK2 activity and collagenase 1 (MMP-1) gene expression. Mol Cell Biol 221:23732383. CrossRef

Ye X, Shobe JL, Sharma SK, Marina A, Carew TJ (2008) Small G proteins exhibit pattern sensitivity in MAPK activation during the induction of memory and synaptic facilitation in Aplysia. Proc Natl Acad Sci USA 105:20511-20516. CrossRef Medline

Zhang Y, Liu RY, Heberton GA, Smolen P, Baxter DA, Cleary LJ, Byrne JH (2012) Computational design of enhanced learning protocols. Nat Neurosci 15:294-297. CrossRef 